yeomen farmers, and for several hundred years had owned and lived at their farm. This is situated in one of the healthiest districts in the kingdom, where the people are notoriously robust and hardy. They have records of several intermarriages amongst relatives, probably brought about by the desire to keep the property in the family. The father and mother were second cousins, and were much above the average in physique and strength. The mother says her grandmother suffered in the same way as these children. There were nine children, five of whom are healthy and four developed this disease.

The eldest child, a girl, was said to commence the disease at the age of 14, and died from it at the age of 26 , being then unable to leave her chair to which she was lifted out of bed. The feet and hands, they say, were deformed in the same way as the two cases that follow.

The eldest son commenced to show symptoms between 13 and 14 years of age. When I saw him he was a man of enormous physique, 6 feet 2 inches in height, and broad in proportion. He was helped from bed every morning into a specially made massive and immovable chair where he remained all day. His mental powers were good, his speech and facial aspect were as described in the first case, and the eyes were normal; there was complete paresis of the legs with great stiffness, and the legs were swollen to an enormous size, with the skin coarse and pitted, almost suggesting elephantiasis. The feet were characteristically deformed, short, stumpy, with high-arched instep, with the toes over-extended at the interphalangeal joints. When I first saw him there were a number of deep excavated foul and painful ulcers in the legs; these, they said, had been caused by slight knocks. They slowly disappeared after prolonged treatment, and as great care was exercised not to knock them there was no recurrence. He said that the least scratch or cut was difficult to heal. The paresis was almost complete in the arms and hands; he could, however, lightly grasp between the index and second fingers; the thumb was entirely useless. The manus cava was well marked. Lateral nystagmus was also present in fixing the eye at an unusual angle. There were no crises, nor neuralgias of any kind. The feet were drawn inwards so that they rested on their outside edges. He died eight zears ago from pneumonia at the age of 42 years.

The next child affected, a girl, showed symptoms first at the age of 14 years. She was able to get about up to the age of 35 , but is now unable to walk or feed herself. She has slight power of movement in the arms and only with great difficulty can grasp a pencil, when it is held up to her, between the index and second fingers. Her feet and hands show the pes cavus and manus cava symptoms in a typical manner. Nystagmus is present. The speech is thick and monotonous and the attitude and facial expression are similar to the cases already described, with well-marked bending of the spine. The eyes are normal and the sight is good. She complains of no pain and says she should be well if she only had the use of her limbs. During the last two years her legs have begun to swell, and the swelling is now well marked, but not extensive, from three inches below the knee downwards. The swelling does not pit easily on pressure and the skin in the affected part is beginning to get coarse, but there are no ulcers.

The second son, although not afllicted with this disease, is mentally deficient. He is, however, a well-grown powerfal man. With this exception, all the children are mentally much above the average and are fine specimens of humanity, one son having a wide reputation as an athlete.

The only case which has been treated for the constitutional disease is the one first described, and in view of the fact that the disease had already existed for nearly eight years and taking into account the age of the patient when treatment was commenced, I think that we may regard the progress of this case as favourable. It is impossible to say that the disease has been arrested ; in fact, in the nystagmus and knee reflexes the disease has made some headway, but, as the history shows, slight improvement has taken place in some respects. Further, the locomotive powers and the use of the arms are quite as good now as they were when I first saw her 11 years ago.

It would be interesting to know the result of continuous treatment of the same kind applied in the early stages of Friedreich's ataxia, especially in young patients in whom active development is still progressing.

The history of these cases strongly supports the theory that the disease is hereditary, and bears out the idea that it may be caused by some error in the developmental forces, which is not accidental and which may possibly arise in some cases from consanguinity. But apart from the interest in the hereditary character of this disease to which this range of cases clearly points, there are some clinical aspects which I have not seen recorded hitherto. CEdema appeared in the three children who had suffered for the greatest number of years and was a symptom which developed late in the course of the disease and which progressed pari passu with the paresis. At the same time the skin also underwent a progressive change, becoming in the first place coarse and pitted and eventually assuming almost the appearance of elephantiasis. In these cases it was associated with trophic ulcers, which, however, were different from the ulcers found in cases of locomotor ataxia and other spinal lesions in that they were markedly painful.

Shelton, Stoke-upon-Trent.

\section{DISPLACEMENTS OF THE SEMILUNAR CARTILAGES.}

By KENELM H. DIGBY, M.B., B.S. LOND., SURGICAL REGISTRAR, GUY'S HOSPITAL.

CertaIN joints in man possess interarticular fibrocartilages, namely, the knees, the temporo-mandibular articulations, the sterno-clavicular joints, and the joints between the bodies of the vertebræ. The triangular cartilage separating the inferior radio-ulnar from the wrist-joint is an additional instance. In each case the interarticular cartilage forms a more or less complete partition, producing a double joint, each part of which has its own distinct movement-a movement which (except in the case of the intervertebral joints) is of a different kind from that of the neighbouring part.

In the knee, on the femoral side of the interarticular cartilages, hinge motion of flexion and extension takes place; on the tibial side a slight degree of rotation is permitted during flexion. In the temporo-mandibular joint a gliding backwards and forwards takes place above the interarticular fibro-cartilage, whilst below the cartilage hinge movements of opening and closing the mouth are possible, together with some little rotation. In the sterno-clavicular joint up-and-down movements are said to occur on the clavicular side, and forward and backward movements on the sternal side of the interarticular cartilage. The triangular cartilage, uniting the radius to the styloid process of the ulna, by its upper surface axially rotates on the ulna during pronation and supination, but its lower surface helps to form the wrist-joint proper at which backward and forward or lateral movements can occur. The spine gains increased range of movement from the duplication of the intervertebral joints by the discs, but the kind of movement in adjacent synovial sacs is similar.

With the exception, therefore, of the intervertebral joints, the variety of movement in a joint is increased by the presence of interarticular cartilage. Should an interarticular cartilage lose its attachment, it is liable to shift its position and interfere with the mechanism of the joint. This is evidenced in the internal derangements of the knee and of the temporo-mandibular articulation. The other interarticular cartilages in man are so securely anchored as to resist detachment. The general rôle of interarticular cartilages has thus been outlined. In the special case of the knee certain points in their structure and function require closer examination.

The general shape of the cartilages is suggested by their name-semilunar. But there are two important points in their conformation which are less frequently emphasised. In the first place, they are wedge-shaped in section, with the apex pointing into the centre of the joint (vide Figs. 1, 2, 3, 4), and so, considering that both upper and lower surfaces are smooth and freely lubricated with synovial fluid, it is difficult to imagine that a normally lubricated, undetached cartilage should become severely squeezed between femur and tibia. In the second place, whilst the lower surface of each cartilage is flat, gliding easily on the fiat top of the tibia, the upper surface is concave, so that each semilunar cartilage forms a socket for one femoral condyle. The oval shape of 
the internal semilunar cartilage is associated with the greater antero-posterior surface of femur in contact with it during complete extension. During flexion the internal condyle of the femur is in close articular contact only with the posterior portion of the internal semilunar cartilage. (Fig. 1.) The base of each wedge-shaped cartilage is planted fairly upon the capsular ligament, the fibres of which pass into the fibro-cartilage.

FIG. 1.

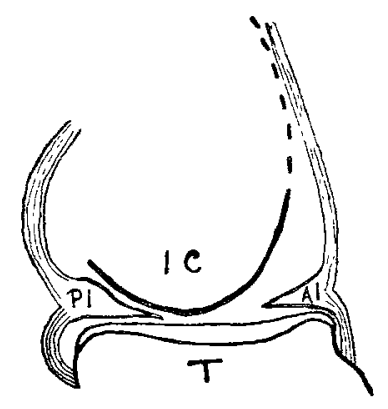

FIG. 2.

FIG. 3.

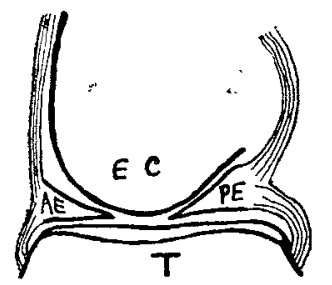

FIG. 4.

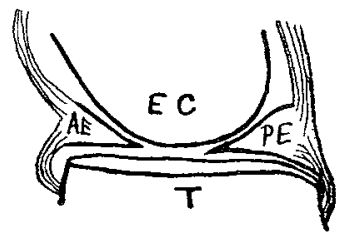

Fras. 1, 2, 3, 4.-I c, Internal condyle of femur。 E c, External condyle of femur. $T$, Upper end of tibia. A I, Anterior part of internal semilunar. P I, Posterior part of internal semilunar. A $\mathrm{E}$, Anterior part of external semilunar. P E, Posterior part of external semilunar.

These figures are diagrammatic sections: 1 . Through the internal condyle in flexion. 2. Through the internal condyle in extension. 3. Through the external condyle in flexion. 4. Through the external condyle in extension.

Note the wedge-shape of the semilunar cartilages in section, their superior concavity, and that the internal condyle only completely occupies the internal semilunar in extension. In flexion it does not touch the anterior end of the semilunar; the ligamentum alarium fills the interval. The diagrams also show the anterior part of the capsule tight and the posterior part of the capsule relaxed during flexion.

The so-called "coronary ligament" is nothing but that piece of the capsule, stretching from the periphery of the cartilage to the tibia, which has a length of about half an inch and upwards. Since the cartilage is attached to the capsule much nearer the tibia than the femur, it follows that it has closer tissue connexion with the tibia than with the femur. And this at first sight is what renders the separation of semilunar from tibia difficult of comprehension. But it must be remembered that if there be no direct anatomical union of femur and semilunar there is, nevertheless, in virtue of the upper concavity of the semilunar a close functional union, inasmuch as the femoral condyles are firmly fixed in the hollows of the semilunars by the weight of the body, atmospheric pressure, and muscular tone. So strong is this union that in excessive rotation at the knee the semilunars tear their capsular anchors before parting company with the femoral condyles with which they have only indirect tissue connexions.

The chief movements at the knee are flexion and extension. Such movements can only take place between the femur and the interarticular cartilages. F or, below the cartilages, the opposed articular surfaces are approximately flat. A peculiar kind of rotation accompanies the last stage of extension and the first stage of flexion. Owing to the greater antero-posterior articular surface of the inner condyle of the femur the completion of extension at the knee is accompanied by a slight rotation outwards of the tibia on the femur. This rotation has the effect of diminishing muscular strain in the erect position; partly because of tightening of all the ligaments; partly because, as hinge movements cannot occur without some rotation taking place, and as, in the erect position there is no tendency to rotation as there is to flexion, only slight tone of the biceps is required to maintain the attitude. But apart from this the flexion and extension are not the movements of a hinge joint for the parts of the femur in articular contact are constantly changing. It is probable that during extension the forward roll of the lower end of the femur (Fig. 5) will tend to sweep any loose body in the front of the joint backwards into its centre, and thus may be explained the presence of a displaced anterior end of the semilunar in the intercondyloid notch, a condition of things sometimes dis-

FIG. 5.

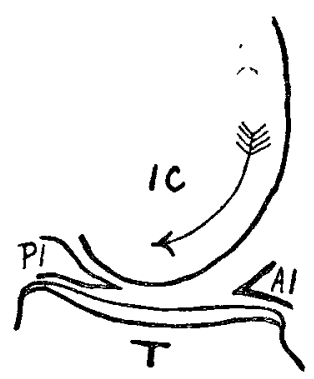

Diagram to show the "roll" of the femoral condyles during extension owing to fresh parts of the femur coming into articular contact. $x \mathrm{c}$, Internal condyle here shown in flexion. T, Upper end of tibia. A I, Anterior end of internal semilunar cartilage. $P$ I, Posterior end of internal semilunar cartilage.

closed at operation. It is absurd to suppose, however, that this tendency could result in detachment of a healthy semilunar cartilage. Moreover, the first detachment of a semilunar cartilage does not occur during sudden extension of the knee.

In addition to flexion and extension anatomists describe a rotation which takes place between the semilunar cartilages and the tibia. I have heard it said that such movement does not occur and that the apparent rotation really takes place at the foot. That there is real rotation of the tibia when the knee is flexed is shown by the alteration of direction of the inner surface of that bone. This is proved also by the fact that, where the foot and ankle are immoveably cased in plaster-of-Paris and the thigh fixed, some abduction and adduction of the foot still occur, and these movements could then only be produced by rotation at the knee. If a springclip with a pointer attached be made to encircle the lower part of the leg where the bones are subcutaneous the degree of rotation can be fairly measured. The range of inward rotation is about twice as great as that of outward rotation.

This rotation below the semilunar cartilages does not occur in the fully extended limb, for then nearly all the ligaments are tight. Indeed, when everything is severed except the crucial ligaments, these are still sufficiently taut to prevent the slightest rotation. (The inability to rotate has nothing to do with the adaptation of bony surfaces in extension, for

FIG. 6.
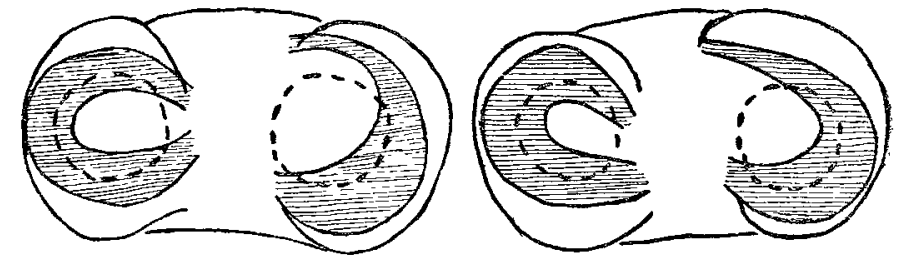

Diagrams to show rotation of the tibia below the semilunar cartilages during flexion of the left knee, as seen from above. The simple outline is that of the upper end of the tibia, the shaded areas denote the semilunar cartilages, the dotted circles are intended to represent (quite diagram matically) the femoral condyles. The left-hand diagram is of a flexed knee without any rotation of the tibia; the righthand diagram is of a flexed left knee showing a slight degree of untward rotation. The front of the joint is above in the diagrams.

in all positions the articulating plane of the tibia is a flat surface.) The rotation during flexion is permitted by the lax state of the ligaments. The mechanism of this rotation can be seen in any dissected joint. Each semilunar cartilage is firmly fixed by its ends to the middle of the joint. Peripherally the capsular attachments are lax. In rotation the tibia rotates beneath the cartilages, so that relatively to the upper surface of the tibia one cartilage swings forwards 
and the other backwards, each, of course, maintained in contact with the corresponding femoral condyle. (Fig. 6.)

Though the range of this rotation is slight its value is considerable. It adds to the fine adjustments of the foot which are required in running over unequal ground, and, what is perhaps more important, must play a great part in checking dislocation of the knee. If it were not for the slight "give" of the semilunars, any sudden wrench during flexion would be sufficient to throw the condyles of the femur off the tibia. Of course, the semilunar cartilages also tend to prevent dislocation by forming a socket, if a shallow one, for the femur. But if they were fixed to the tibia as the cotyloid ligament is fixed to the acetabulum, a sharp twist would still risk dislocation.

Outward rotation of the tibia at the knee is produced normally by the biceps muscle, inward rotation by the popliteus and inner hamstring muscles-viz., sartorius, gracilis, semitendinosus, and semimembranosus. Abnormally severe twists are caused by indirect violence usually applied to the foot, and there is detachment of the semilunar from the capsule and so from the tibia. These severe forms of violence are considered subsequently.

Inward rotation of the tibia is checked by a "winding up " of the crucial ligaments. Outward rotation is first checked by the attachment of the anterior end of the internal semilunar cartilage to the front part of the capsular ligament. Thus it is upon this portion of the attachment of the periphery of the internal cartilage to the capsule that chief strain is likely to fall. The action of the crucials is well seen if a knee-joint be denuded of all its ligaments except the crucials, and be flexed to a right angle. The tibia then can only be rotated inwards some $20^{\circ}$, but can be rotated outwards through two right angles so that the anterior border looks almost directly backwards (Fig. 7).

FIG. 7.

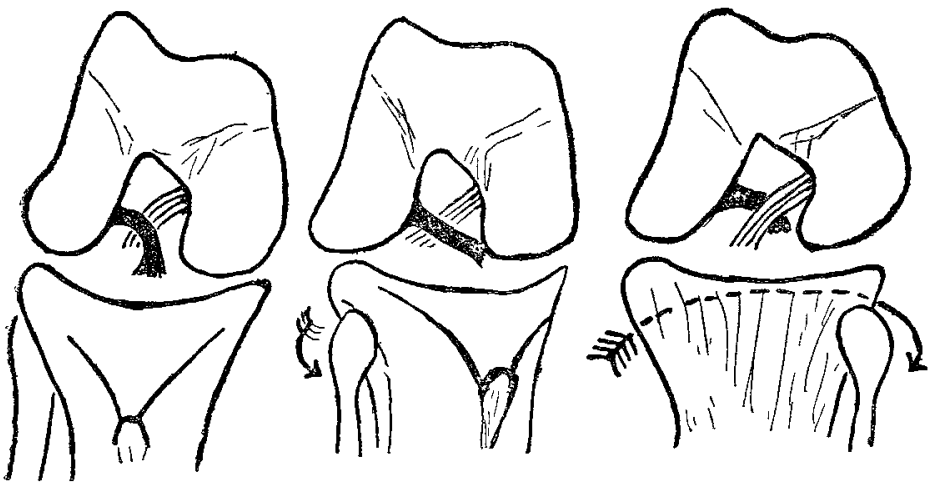

Three diagrams of the flexed knee-joint to show the action of the crucial ligaments in rotation of the tibia. .11 the other ligaments have been divided. In the left-hand diagram there is no rotation. The middle diagram shows inward rotation of the tibia checked early by winding up of the crucial ligaments. The right-hand diagram shows outward rotation continued through nearly two right angles, so that the back of the tibia is now in front. Outward rotation of the tibia first unwinds and relaxes the crucial ligaments. Hence these interosseous ligaments check inward, but not outward, rotation. The anterior crucial is black, the posterior crucial is lined in the diagrams.

Strain does not come upon the attachment of the posterior ends of the semilunar cartilages to the capsule, because rotation only occurs during flexion of the joint and the posterior part of the capsule is then relaxed and can follow either cartilage forwards, as opposed to the anterior fibres of the capsule which are tense, especially if the vasti be acting.

The semilunar cartilages are composed of fibro-cartilage. Cartilage is the one tissue in the body which can stand extreme pressure and friction. So that the laceration and pathological changes seen in displaced semilunar cartilages can hardly be due to squeezing between tibia and femur. If they were, since action and reaction are equal and opposite, one might expect the adjacent articular cartilage of the femur and the tibia to show similar changes, which, I believe, they do not in any recent case. The blood-supply of the semilunar cartilages comes through their peripheral attachments to the capsule; when a cartilage is detached its blood-supply is interfered with and it undergoes degenerative changes and may then be secondarily affected by pressure between femur and tibia. The smoothness of surface is lost, degenerative changes may follow, and the whole mass if sufficiently detached may be carried by the "roll" of the femur into the centre of the joint. I suspect that the pain of "slipping of a cartilage" is due to pull upon, and laceration of, the capsular attachments and not to any "squeezing" of a probably' insensitive fibro-cartilage. Any initial crushing of a cartilage, the attachments of which have not been lacerated, is well-nigh inconceivable.

Certain facts appear promirently in the histories of cases of internal derangement of the knee. The injury is rarely due to direct violence. The patient has often fallen, but the fall is the result rather than the cause of the injury. It is usually the indirect application of force which determines the lesion. The knee at the moment is almost always in some position of flexion, the quadriceps extensor muscle is often contracting, and commonly the weight of the body is being transmitted partly through the articulation. But the exciting force is a rotary one, either a twist of the foot when the thigh is more or less fixed, or a twist of the femur as the whole body swings round whilst the foot is, for instance, firmly planted on the ground. These points are illustrated in most careful accounts of the accident. The following are some of the usual methods of meeting with the injury. They have been taken from some of the reported cases and also from an examination of 100 consecutive cases which were operated upon at Guy's Hospital up till the end of 1907.

1. A slip or twist whilst running, walking, or dancing. rhe wrench at the knee, especially in recurrent cases, is often of a trivial nature.

2. Catching the inner side of the fore part of the foot against some obstruction, or catching the foot in a hole, or between two planks.

3. Turning suddenly on one leg, as in dodging a vehicle in the street, or as in football, golf, cricket, tennis, or gymnastic exercise.

4. Slips or short falls on to one leg, (with the foot in the abducted position), stepping off a platform on to the permanent way, or stumbling downstairs. The patient often describes his falling with "one leg doubled under him."

5. Being tripped up as in wrestling or football.

6. Attempting to rise after squatting some time upon the toes or kneeling with toes turned out.

7. Turning over in bed, the foot being restrained by the weight of the bedclothes. This is by no means so rare as might have been expected. A young woman, aged 21 years, " turned round in bed to stop an alarm clock and felt something go in her knee and the knee began to swell." Considerable displacement of the internal semilunar cartilage was found at operation.

8. Climbing a ladder or going upstairs, the former especially necessitating outward rotation at the knee.

9. Kicking at a football with the inside of the fore part of the foot, chiefly in the case of one unaccustomed to play. 14 of the 100 cases collected from the Guy's Hospital records were ascribed to football. Most of these would come under the headings 3 and 5 .

10. The foot may be caught in some moving structure, as in the spokes of a revolving wheel.

11. A small number of cases have been reported as the result of direct violence. Except for these few cases nearly every accident was due to a twist or wrench.

It will be seen that whether the foot is twisted or whether merely fixed whilst the body rotates, an important factor is the leverage afforded by the projection of the foot at right angles to the leg. Man pays for the privilege of being a plantigrade animal by his liability to injuries of the lower limbs by rotary violence. The outward twist of the foot exerts a more powerful strain than does the inward. For to turn the mass of the body by twisting the foot outwards requires more force than to do so by turning the foot inwards. Moreover, the usual attitude of out-turned toes invites the outward rather than the inward twist. Most of the indirect injuries of the lower limbs are due to this outward twist: Pott's fracture-dislocation (with it spiral fracture of the

1 Quain's Anatomy, 1891, vol. i., Part II., p. 250, under heading of "Fibro-cartilages": "No nerves have been traced into any of th cartilages, and they are known to be destitute of sensibility." Moreover, in a case of displaced internal semilunar cartilage, in which com plete extension of the knee was impossible, owing (as disclosed at operation) to part of the internal cartilage lying between the ends of the bones, the patient could bear his whole weight upon that leg without pain, and considerable force could be painlessly expended in attempts to straighten the knee. 
fibula, so clearly demonstrated by Mr. W. Arbuthnot Lane), spiral fracture of the bones of the leg (where the fibula in consequence breaks at a higher level than the tibia), the usual displacement of the internal semilunar cartilage, spiral fracture of the shaft of the femur, and intracapsular fracture of the neck of the femur. The particular lesion which follows the outward twist of the foot is determined by the position of the rest of the limb, the age of the individual, and like factors.

In a careful investigation of the clinical histories it will be found that many semilunar displacements are certainly due to outward rotation, which is what one would expect; for the position of the foot invites outward rotation, outward rotation imposes a greater strain, and it is not checked by the crucial ligaments as is inward rotation.

Sir William Bennett has described exactly the pathological anatomy of internal derangements of the knee occurring in his large surgical experience of these cases. He finds the larger proportion involve the anterior end of the internal semilunar cartilage, the anterior two-thirds being more or less torn from the capsule or even the anterior end of the cartilage also detached. Whilst this is the cummon condition, injuries of the posterior part of the internal semilunar and also injuries of the external semilunar cartilage occur in a proportion of cases. Some of the lacerations of the posterior part of the internal semilunar cartilage found in addition to partial detachment are possibly produced during the removal of the cartilage, since they may be mimicked in removing normal cartilages post-mortem.

The common semilunar displacement is due to violence applied to the foot usually in an outward direction. It is difficult to give statistical proof for this statement as accounts of the accident rarely state whether the "wrench" was outwards or inwards. (It must be admitted that the patient sometimes states that the foot was turned inwards at the first accident.) The tibia rotates outwards beneath the semilunar cartilages which cap the femoral condyles. As the knee at the time is flexed there are no tibio-femoral ligaments which can limit outward rotation of the tibia. The strain may be expected to fall upon the capsular attachments of the anterior end of the internal semilunar and the posterior end of the external semilunar cartilage. As the posterior part of the capsule is relaxed it can follow the external semilunar into the joint. But the anterior part of the capsule is taut and the internal semilunar cartilage adhering to the femoral condyle is dragged from the capsule in front (Fig. 8) and shoots with a click into the joint.

FIG. 8.

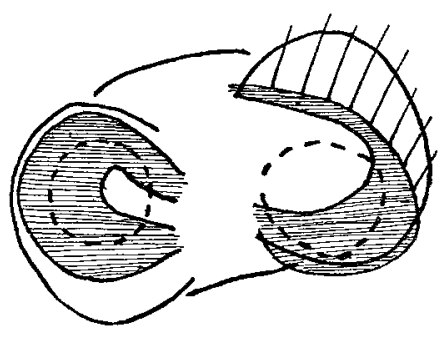

\begin{abstract}
Diagram to show excessive outward rotation of the left tibia below the semilunar cartilages during flexion of the knee, as seen from above. The simple outline is that of the upper end of the tibia, the shaded areas denote the semilunar cartilages, the cotted circles are intended to represent (quite diagrammatically) the femoral condyles. The lines radiating from the anterior two-thirds of the periphery of the interna semilunar cartilage mark the region of greatest strain. Compare the two diagrams in Fig. 6. The front of the joint is above in the diagram.
\end{abstract}

Even the anterior cornu may be detached and the loose part be rolled into the intercondyloid notch; or the periphery of the cartilage may remain on the capsular ligament and only the inner margin be torn away; or, the anterior end of the cartilage maintaining its hold, the femoral condyle may subsequently ride over the peripherally detached cartilage, which will then turn over into the joint like the " handle of a bucket," as it bas been aptly described.

During flexion any loose portion has room to lie free in front, but the greater antero-posterior length of the fully extended internal condyle can spare no room, so that unless the displaced cartilage be restored to its normal position complete extension cannot occur, and the so-called "locking" of the knee ensues. (Fig. 9.) Short of complete rupture of the peripheral attachment there may be laceration with hæmorrhage. This may give rise to a loose body in the joint, or even by its size may push the semilunar cartilage inwards, preventing extension, and, as Sir William Bennett pointed out, only slowly absorbing, the condition is not susceptible of manipulatiory reduction. Laceration of the capsular attachment will give rise to the sickening pain, the local tenderness, and the effusion into the joint. Complete extension may be impossible. Its attempt does not always give rise to severe pain. The relaxed condition of the ligamentum patellæ first described in Hey's original paper

FIG. 9.
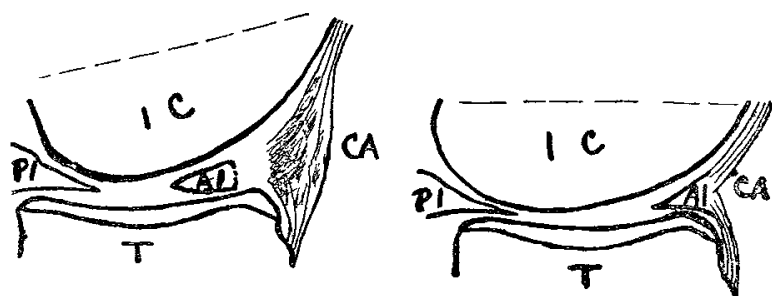

Diagrams to illustrate incomplete extension of the knee in a case of displacement of the fore part of the internal semilunar cartilage. I C, Internal condyle of femur. T, Upper end of A $r$. Anterior end of internal semilunar cartilage. $C$, Anterio part of capsule.

The right-hand figure shows normal complete extension. The The right-hand figure shows normal complete extension. The left-hand figure shows the capsule lacerated and slightly swollen, and the anterior end of the internal semilunar cartilage separated from the capsule and displaced inward into the joint. Note how the slight elevation of the anterio part of the upper surface of the inner tuberosity of the tibia tends to prevent the anterior end of the internal semithe knee after displacement has once occurred.

may signify relaxed tone of the quadriceps, and so diminution of tension upon the lacerated capsule. More often, perhaps, the relaxed condition is the result of repeated effusions into the joint. Strong outward rotation of the tibia gives rise to pain over the peripheral attachment of the internal semilunar. Strong inward rotation is painless. But if the knee is locked it may be impossible to produce any rotation.

It is easy to see how conservative treatment may sometimes result in benefit. The cartilage, if displaced, is reduced by manipulation; its base lies in contact with the lacerated capsule, and, if all rotatory movements are avoided, adhesions will form. The subsequent use for some months of an Ernst apparatus, which prevents the least rotation at the knee whilst permitting flexion and extension, may result in a cure. The wearing of a boot with sole and heel higher on the inside has been employed to favour adduction and inversion of the foot. The avoidance of exercises, such as football and hockey, which involve twisting on the legs, is imperative. The patient is told to walk with the toes straight in front and not turned out.

The normal and pathological anatomy, the clinical histories and symptoms, the conservative methods of treatment which occasionally meet with success, all favour the view that excessive outward rotation of the tibia beneath the interarticular cartilages produces a laceration of the capsular attachments of the fore part of the internal semilunar cartilages which is the usual lesion in this class of injury.

I wish to express my indebtedness to Mr. G. F. Stebbing for his valuable advice and criticisms in connexion with this paper.

Hospital for Sick Children : Post-Graduate CLASSES. - The winter session of lectures and demonstrations at the Hospital for Sick Children, Great Ormond-street, commenced on Jan. 13th, when Dr. A. E. Garrod demonstrated selected cases from the medical wards. On Jan. 20th Mr. E. M. Corner will treat of Appendicitis and its Treatment in Children. The lectures are given at 4 P.M. on each Thursday and are free to all qualified medical practitioners. 


\section{A CASE OF SEPTIC INFECTION CONSE- QUENT ON MASTITIS.}

BY ALEXANDER D. PITHIE, L.R.C.P., L.R.C.S. EDIN., L.F.P.S. GLASG.

THE patient in the following case was a married woman who was confined prematurely of an eight months' child on June 4th, 1909, and made a fair recovery. Her highest temperature during the first month was on the fifth day, when it was $99^{\circ} \mathrm{F}$., with a pulse-rate of 102 . In the right breast she had no milk, and in the left only a little, but not enough for the child, who accordingly had to be fed with the bottle. The left breast ached from the time the child was put to it till the time of sitting up, when it felt so heavy that the nurse supported it with a sling on the tenth day, after which it was more comfortable. At the end of the first month the patient menstruated, when the left breast became very tender and ached, a lump appearing which was so tender to the touch that she could only lie on her back. The district nurse then advised hot fomentations for four days, when the pain became less, but when the next period came on the pain was worse, and at the time of the third period, or about Sept. 4th, there was found a lump of the size of a walnut, which became inflamed and shiny, forming an abscess, which broke on Sept. 25th, and offensive thick yellow pus was discharged. She still continued the fomentation for ten days. The nurse saw her on Oct. 5th, and kept her in bed; the temperature was normal, but the patient was very weak and on that night she had a rigor, with sickness and faintness.

When called to see the patient on Oct. 7th I found her in an almost collapsed condition, with a temperature of $103^{\circ} \mathrm{F}$. and a pulse-rate of 100 , the temperature rising that night to $104 \cdot 8^{\circ}$. The breast was red but not swollen, the redness being more of a skin rash. To the left of the breast there was an opening which was evidently superficial, as a probe could not be inserted to any distance, but no evidence of pocketed pus could be found. No other condition to account for the high temperature could be detected. Strychnine and quinine were administered and the temperature gradually came down till the 11th when it suddenly rose again to 105. This time not only the breast but also the whole of the chest and upper part of the abdomen became covered with the rash which was of a bright red colour. The right breast also became painful and a painful spot appeared on the left shoulder. No evidence of suppuration was to be found. The patient at this time had the appearance of a person in extremis. I then injected 10 cubic centimetres of Allen and Hanburys' polyvalent antistreptococcic serum in the walls of the abdomen, repeating the injection in ten hours. As the vomited I decided to give it per rectum, which was carried out for the remainder of the treatment. For the first three days she retched after each dose of serum, but then tolerance was established and the nausea passed off. Administration of serum was carried out three times a day until the 15th when it was given twice daily, and then once a day till the 18th, when it was stopped, the temperature being normal and the pulse-rate 88 . Her condition otherwise was very good and all appearances pointed toward a sure convalescence. On the 26th, however, the temperature once more began to increase, being $100 \cdot 2^{\circ}$ in the morning and rising to $102 \cdot 4^{\circ}$ the next morning, when a small superficial lump was found on the left breast. Again the whole of the chest and neck was covered with the rash. On the 27th serum was given twice, and on the following day three times, when the temperature came down to $97 \cdot 80^{\circ}$, serum being given once daily and for the last time on the 30th. The patient then made a slow but satisfactory recovery, and is now quite well.

The points of interest in this case are the evident absorption of septic matter from a mastitis without any evident collection of pus; the prompt and satisfactory action of the serum after quinine had been given without good effect; and, finally, the recurrence of the symptoms in a few days after the serum had been stopped, and the controlling power of the serum when again resorted to. This leads me to think that in similar cases it would be well to continue the administration of serum for some time after all symptoms had subsided, if even in smaller or less frequent doses.

Lymington, Hants

\section{attedical Sociuties.}

\section{ROYAL SOCIETY OF MEDICINE.}

\author{
MEDICAL AND SURGICAL SECTIONS. \\ Duodenal Uloer.
}

COMBINED meetings of these sections were held on Dec. 14th, 1909, and Jan. 11th, Mr. G. H. MAKINS being in the chair.

Mr. B. G. A. Mornihan delivered the opening address on Duodenal Ulcer, which was printed in THE LANCET of Jan. 1st, p. 20

Mr. H. Colminson read a paper on the Analysis of 197 Cases of Duodenal Ulcer operated upon by Mr. Moynihan (1900-1908).

Sir T. LAUDER BRUNTON said that with regard to the season it had been his experience that gastric and duodenal ulcer-he could not distinguish them in his list of cases-was much more common in two periods of the year. He had not noticed it so much in mid-winter, but much more in autumn and spring. And the reason which he had assigned to himself was that people put of too long their winter clothing and so were chilled in autumn, and they put off their winter clothes too early in the spring, and in each case a chill was liable to result. Many years ago, when he was working at the physiology of the subject for Burdon Sanderson's "Hand-book for the Physiological Laboratory," he made very many experiments upon the stomach and upon the intestine. He tried, and in vain, to make gastric ulcers. He put a ligature round a small portion of the mucous membrane of the stomach which he had previously opened, and then closed the wound, keeping the animal under narcosis, and allowed it to remain four or five hours. He thought that at the end of that time an ulcer would be found at the spot where the blood-supply had been stopped by the ligature. But in none of the cases was he successful. Mr. Moyniban had left out reference to one part which he (Sir Lauder Brunton) would like to supplementnamely, the cause of the pain. The pain came on between two and four hours after the stomach had commenced to discharge its acid contents. Those acid contents came over the duodenum and smarted the ulcer, causing intense pain by the acid acting on the bare surface. This pain could be relieved by not putting anything into the stomach which causes a reflex contraction of the pylorus, the acid juice from the stomach was thus prevented from flowing into the duodenum, and any acid which was already there was quickly neutralised by the alkaline juice of the duodenum itself, possibly partly by some of the bile. That might be imitated by giving bicarbonate of soda in large doses. Time was a very important factor in the diagnosis, as $\mathrm{Mr}$ Moynihan had said. Sometimes one might be misled as to the nature of the lesion, and yet be right as to its position. In one case which was brought to him the symptoms were pain four hours after feeding, and he thought there must be something wrong in the duodenum, but it did not yield in the ordinary way to the administration of alkalies, and he said he was doubtful as to whether it was ulcer or not, but he thought it must be something wrong with the duodenum. He never saw the case again. Eighteen month afterwards there was a description in THE LANCET of an operation on that patient. From that it turned out that he was right as to the position of the lesion, but it was a kink and not an ulcer. Time had also been very valuable to him in diagnosing duodenal ulcer when the diagnosis for a time would have been very doubtful. As an example he might mention that he was once asked to see a patient who was suffering from severe lumbago. On examination he found that the so-called lumbago was limited to the right loin; it did not extend round the back. It came on between two and three hours after a meal and was again stopped by food. He (Sir Lauder Brunton) concluded that it was a duodenal ulcer, and told the doctor that it could be perfectly well ascertained whetber it was duodenal ulcer or not by giving a large quantity of bicarbonate of soda, and if it was duodenal ulcer, by neutralising the acid of the gastric contents one would stop the pain. The doctor tried it and the pain ceased immediately, so that there was some doubt as to the 